\title{
Dissemination Strategies: How Do They Influence the Uptake of New Three Rs Methods Across Laboratories and Other Boundaries?
}

\author{
A Report of a Workshop held by the European Partnership for Alternative \\ Approaches to Animal Testing (EPAA) Working Group 3a
}

\begin{abstract}
Sally Robinson, ${ }^{1}$ David Basketter, ${ }^{2}$ Rita Bussi, ${ }^{3}$ Joachim Coenen, ${ }^{3}$ Philip Connolly, ${ }^{4}$ Signe Gry Elvig-Jørgensen, ${ }^{5}$ Helma Hermans, 6 Alan Hoffmeister, 7 Antonio Lacerda, ${ }^{8}$ Jens P. Linge, ${ }^{9}$ Horst Spielmann, 10 Michael Török, ${ }^{11}$ Jan van der Valk ${ }^{12}$ and Tim Watson ${ }^{13}$
\end{abstract}

${ }^{1}$ AstraZeneca R\&D Alderley Park, Safety Assessment UK, Macclesfield, Cheshire, UK; 2 DABMEB Consultancy Ltd, Sharnbrook, Bedfordshire, UK; ${ }^{3}$ Merck KGaA, Darmstadt, Germany; ${ }^{4}$ Corporate Communications, Merial, Lyon, France; ${ }^{5}$ Novozymes A/S, Bagsvaerd, Denmark; 6 Scientific Writing \& Consultancy, Alicante, Spain; "Charles River UK Limited, Margate, Kent, UK; ${ }^{8}$ European Commission DG ENTR Brussels, Belgium. ${ }^{9}$ ECVAM IHCP JRC, Ispra, Italy; ${ }^{10}$ BfR - Federal Institute for Risk Assessment, Berlin, Germany; ${ }^{11 D S M}$ Nutritional Products Ltd, Basel, Switzerland; 12Netherlands Centre Alternatives to Animal Use (NCA), Department of Animals, Science \& Society, Faculty of Veterinary Medicine, Utrecht University, The Netherlands; ${ }^{13}$ NC3Rs, London, UK.

\section{Preface}

The European Partnership for Alternative Approaches to Animal Testing (EPAA; 1) organised a workshop in October 2007, to review whether dissemination plays a role in the successful uptake of new Three Rs methods. The main conclusions from the workshop are summarised in this report.

There is probably sufficient awareness about the need for the Three Rs, due to societal and legislative pressure and industry requirements. Also, there are many opportunities for gaining funding for research, and an abundance of organisations to provide information on the Three $\mathrm{R}_{\mathrm{s}}$, although these tend to work in isolation. The EPAA workshop identified a gap. This was the absence of a process and/or organisation/institution supporting the postvalidation and implementation of new methods that would help ensure the rapid and widespread uptake of new alternative methods.

The case studies presented at the workshop have clearly illustrated that what has been achieved so far is mainly due to the resource and perseverance of personal champions. This is not a very efficient way forward for the future.

Since dissemination plays an important role during the post-validation/implementation phase and for regulatory acceptance, the EPAA will see whether the synergy between the dissemination and validation and acceptance work strands can be improved, and will ensure that the regulatory authorities are consulted.

\section{Introduction}

The principles of the Three $\mathrm{Rs}_{\mathrm{s}}-$ Replacement, Reduction and Refinement - were originally developed by William Russell and Rex Burch, and are now widely accepted internationally as criteria for humane animal use in research and testing (2). The European Partnership for Alternative Approaches to Animal Testing (EPAA) is an unprecedented collaboration between European Commission (EC) services, trade federations and major companies from seven industry sectors. The partners have committed to pooling knowledge, research and resources to accelerate the development, validation and acceptance of alternative approaches to safety testing, over an initial five-year period. An action programme to promote change has been agreed and is available on the EPAA website (1). As part of the action plan, Working Group 3 (WG3) is addressing the identification, dissemination and implementation of best practice in the use of the Three Rs. Although there is widespread acceptance of the con-

Address for correspondence: Sally Robinson, AstraZeneca R\&D Alderley Park, Safety Assessment UK, Mereside, Alderley Park, Macclesfield, Cheshire SK10 4TG, UK.

E-mail: sally.robinson@astrazeneca.com

aThis document represents the agreed report of the participants as individual scientists. 
cept of the Three $\mathrm{Rs}$, communication, consistency and implementation of current best practice in their application can be further improved.

A large number of organisations have a role in promoting the Three $\mathrm{Rs}$ at different levels in Europe, yet it is evident that the promotion, dissemination, and implementation of the Three $R s$ could be improved. The activities of the different organisations appear to be fragmented, with different remits, scope, funding and levels of impact. In early 2007, Working Group 3 of the EPAA conducted in depth interviews with several Three Rs organisations. The purpose of these interviews was to identify what contributed to successful strategies for dissemination, and what barriers there were to the successful uptake of Three $R \mathrm{~s}$ activities. The organisations interviewed included:

- UK National Centre for Replacement, Refinement and Reduction of Animals in Research (UK NC3Rs);

- Fund for the Replacement of Animals in Medical Experiments (FRAME);

- Centre for Documentation and Evaluation of Alternatives to Animal Experiments (ZEBET);

- Belgian Platform for Alternative Methods to Animal Testing (BPAM);

- European Consensus Platform for Alternatives (ECOPA);

- Association for Assessment and Accreditation of Laboratory Animal Care (AAALAC);

- European Centre for the Validation of Alternative Methods (ECVAM);

- Czech Platform for Alternatives (CZECOPA);

— Italian Platform on Alternative Methods (IPAM);

- Spanish National Platform on Alternatives (REMA); and

- Centre for Alternatives to Animal Testing (CAAT, USA).

The interviews were analysed by WG3. Several factors considered critical for success were identified. Some of these are considered to be outside of the control of those wanting to disseminate new Three Rs methods (e.g. points 7 and 8):

1. Science-driven approach; evidence-based, peerreviewed publications and methods;
2. Clear focus on specific key areas, realistic tiered strategy;

3. Availability of a champion, i.e. someone in a position to push and promote the new method and to provide post-validation support and follow up during implementation;

4. Tangible benefit to those making the change in current practice;

5. Metrics/Key Performance Indicators/visible outputs are helpful, e.g. monitoring of currentlyused tests and new methods, animal number statistics;

6. Good communication tools, e.g. user-friendly website, newsletters;

7. Government policy, e.g. long term support and funding for the Three Rs; and

8. Level of societal concern; high levels of concern promote the Three Rs.

To further explore the validity of these points, a workshop took place on 1-2 October 2007 in Lyon, France, where they were further developed in the light of several case studies. Speaker presentations from this meeting, are available on the EPAA website (1).

\section{Results and Discussion}

\section{General dissemination strategies}

In this document, we aim to summarise the discussions on general dissemination strategies and the case studies highlighting the key points relating to dissemination. Points are highlighted, that confirm any of the eight points (see above) identified from the in-depth interviews with the Three Rs organisations.

When planning how to disseminate a new alternative method, the first step is to identify the audiences that need to know about the method. How dissemination is conducted can have an impact on the chances of success, and the most important part of this is deciding what message to disseminate. This includes considering what will convince each of the audiences to change their current practice and to adopt the alternative method instead. Just stating that a particular method is better is unlikely to work, so identifying, for example, potential concerns and addressing them upfront, could be helpful.

Just as the messages should be tailored to the audience, so should the means of communication. For a broad reach and impact, electronic communi- 
cation forms, such as e-mail and websites, will be most effective. However, for a small, discrete audience, a leaflet or other printed materials may work better. To reach an unfamiliar audience, how they usually receive information should first be established. This will help them in making a decision, and could bring up routes that would not otherwise have been identified.

A timetable should be devised of when and how each audience will be targeted. Key conferences and upcoming policy discussions that relate to the alternative method will need to be factored into the plan, in order to have the maximum effect and influence. Another key decision will be when to start disseminating. The earlier in the process of validation and regulatory acceptance that the dissemination starts, the more involved the audiences will be in the overall process. This must be weighed up against the risk that a certain audience (e.g. a particular regulatory authority) will assume ownership in a way that becomes detrimental further along the process. Repetition helps in getting the message across, so using the same route multiple times to reach a particular audience, or multiple routes single times, is recommended.

Once dissemination has started, its effectiveness needs to be evaluated, in order to decide whether the current methods are working, or whether new ideas need to be tried. Setting targets (e.g. ' $x$ ' companies to be using a new alternative method within 12 months) can help, but these can be difficult to measure. If the aim is to change opinion, a survey of the audience will help to indicate whether the dissemination has been successful. In this context, the development of the ECVAM DataBase service on ALternative Methods to animal experimentation (DB-ALM), implemented on the basis of a precise requirement of the EC and European Parliament, already represents a first step in this direction, such as to enhance the dissemination of information on alternative methods at any stage of development and/or validation $(3,4)$. The DB-ALM in fact provides information on various aspects of animal alternatives (method description summaries; protocols with step-by-step descriptions for the performance of methods in laboratories; details on validation studies; test descriptions and results; information on persons and institutions active in the animal alternatives field), and already counts over 1000 registrations from 64 countries.

\section{The Local Lymph Node Assay (LLNA) as a case study}

The LLNA offers both refinement and reduction as a skin sensitisation method versus the traditional guinea-pig method. OECD testing guideline (TG) 429 describes the LLNA for use in the prediction of sensitisers and non-sensitisers (5). The development and validation of the LLNA took about 15 years, after its initial development in 1987. By 1992, OECD TG 406 had been approved (6). The LLNA was accepted as a screening test, i.e. positive results were accepted, but negative results required further testing. In 1999, the International Coordinating Committee on the Validation of Alternative Methods (ICCVAM) approved the LLNA as a validated method; the ESAC statement followed in 2000. In 2002, OECD TG429 was published, which approved the LLNA for use in the prediction of sensitisers and non-sensitisers. Some relevant points from the LLNA case study are listed below:

1. Any alternative method needs to start with a solid base in science, e.g. the LLNA measures the induction of sensitisation as a function of cellular proliferation in lymph nodes draining the site of topical application of the test substance.

2. At all stages of research and validation (and post-validation), the work should be published. For example, at the time when the LLNA was presented to ICCVAM, there were approximately 60 publications relating to the LLNA. The profile of new alternative methods should be high, e.g. book chapters and reviews, which should be published in a variety of different journals to reach different audiences. Training workshops should be arranged.

Less favourable or inconsistent results should also be communicated, to stimulate an open and clear discussion about a method's limitations. It should always be considered whether any new data have an impact on the method, and work should be ongoing to evolve and improve the method. It is important to win the scientific community over, but it should be recognised that 'not invented here' remains a problem.

3. It is essential to remember that dissemination does not stop after validation. There is a huge amount of work to do post-validation, that could be considered in a similar vein to post-sales support or post-market surveillance. It is beneficial to continue to publish as widely as possible. As the use of a new assay increases, post-validation support should help to avoid having assays/ methods 'rubbished' due to errors, often because of their performance by inexperienced users. This needs to be dealt with effectively, if the uptake of a new method is to be successful.

4. As there is no formal support or process to ensure that post-validation activities run smoothly, successful implementation relies on long-term champions. In the case of the LLNA, these were David Basketter, Ian Kimber, and Frank Gerberick. The champions may be required to take the initiative at several stages, e.g. in the drafting of protocols for regulatory authorities. 
5. It would aid the validation process, if the various authorities worked to the same set of standards and agreed with each other.

In the second part of this case study, data on the actual use of the LLNA were discussed. At ECVAM, the new chemicals database had been searched to follow up on the number of chemicals that had used the LLNA since 1998. There are 4573 chemicals in the database. Of these, 3386 were tested for skin sensitisation, and of these, 56 were tested with the LLNA (as of February 2007, when the new chemicals database was searched). This number may suggest a low uptake of the assay, despite the successful adoption of the LLNA into an OECD test guideline, and the extensive dissemination leading up to (and since) its adoption. However, there are several reasons for this. Until 2002, the LLNA was only approved for identifying sensitisers. Thus, it is unlikely that the assay would have been used as first choice up to this date. In addition, the time taken to complete a registration package of a new chemical will mean that many of the chemicals developed in this time period may still not appear on the register. The guinea-pig assay is still an option, because of the OECD TGs, so it is not illegal to carry it out and it may still be favoured by some companies. This may soon change, as, in the technical annexes of the REACH regulations, for example, it has been stated that the LLNA should be used, unless there is a compelling reason to use another test. However, the regulatory authorities may each define their own criteria for 'compelling reasons'.

\section{The Limulus Amoebocyte Lysate (LAL) bacterial endotoxin test (EndoSafe) as a case study}

Tests have been in use for over 60 years to screen new medicines for the presence of bacterial endotoxins. These initially involved the use of rabbits in a test where the animals were administered the product, and their temperatures were subsequently measured every 30 minutes for three hours. The LAL test uses horseshoe crab blood to detect the presence of bacterial endotoxin. The extract obtained from the blood contains a natural enzyme clotting system that will clot in presence of the endotoxin. Original publications relating to the LAL method stem from the late 1960s (7). The sensitivity of the LAL test is less than 1 part pyrogen/trillion, so it is considered to be an extremely sensitive assay.

1. The LAL test is based on a sound scientific approach and there are numerous publications to support this.

2. The LAL test is reliable, simple, extremely sensitive and quick, and therefore offers many advantages to those who were originally required to make the change from rabbit pyrogen testing. The LAL test allows for real data to be generated on a time scale compatible with process plant activities.

3. This did not involve a validation process, but $a$ weight-of-evidence approach. For example, the Baxter report compared 143196 LAL tests with 28410 rabbit pyrogen tests.

4. LAL testing represented a good commercial opportunity and had an entrepreneurial champion. James Cooper founded a company in 1987 that was acquired by Charles River Laboratories in 1994. Charles River have improved the test as a commercial product, by adding robotics, LAL software, and cytokine stimulation.

5. A commercial marketing approach was used, including decisions on what journals to publish in, what fairs to visit, and what training and support to provide.

6. Regulators, e.g. the US Food and Drug Administration (8), were involved early on.

\section{The 3T3 Neutral Red Uptake (NRU) phototoxicity test as a case study}

If a chemical becomes activated following exposure to UV light, it has the potential to cause phototoxic reactions. In the $1990 \mathrm{~s}$, there was no TG at the OECD level for an animal test for phototoxicity. At that time, it was proposed that an in vitro model should be developed, and this was undertaken as an ECVAM-COLIPA-ZEBET project, co-ordinated by ZEBET. Twenty test chemicals, for which human data were available, were used in all the assays being evaluated. All the assays used the same UV source and UV meter, and a Photo Irritation Factor (PIF) was used to express phototoxicity. A number of mechanistic assays, commercial assays and growth inhibition assays were evaluated. Only the 3T3 test was sufficiently discriminatory, simple, robust and reproducible (9-11). In 1998, a statement endorsing the scientific validity of the 3T3 NRU Phototoxicity Test was issued by the ECVAM Scientific Advisory Committee (ESAC). However, since there was no straightforward process for getting methods accepted at the OECD level, it was not until 2004, six years after the ESAC statement, that TG 432 (12) was endorsed by the OECD Member Countries.

1. Without the active, continuous involvement of ZEBET, the 3T3 test would not have been accepted at the OECD level, thus highlighting the importance of a champion. 
2. The potential in vitro tests were only compared to human data, never to animal data. High-quality human data were therefore the key to success.

\section{Conclusions}

The work of WG3 has shown that dissemination of information on the Three Rs has a direct impact on moving Three Rs methods from R\&D to validation, acceptance and implementation. The eight factors which were identified in the interviews with the chosen Three Rs organisations, were all mentioned as important during the workshop, apart from the perceived necessity of metrics (point 5). Metrics, such as the numbers of animals used in a specific test, can be used to monitor the uptake of new Three $\mathrm{R}$ methods. The value of 'metrics' was discussed, and examples given of situations where metrics can provide impetus for change - for example, a pharmaceutical industry initiative challenging the requirement for acute toxicity studies in drug development (13). The companies involved in this initiative monitored the numbers of acute toxicity studies conducted, and the numbers of animals used in the acute toxicity studies, during the development of a new medicine over a four-year period. The trend toward reduction in the number of studies and in animal numbers, provided the impetus for other companies to change the way they conducted acute toxicity testing.

There is probably sufficient awareness about the need for the Three Rs, due to societal pressure, legislative pressure and industry requirements. Furthermore, there are many opportunities for gaining funding for research and many organisations to provide information on the Three $\mathrm{Rs}_{\mathrm{s}}$, although these tend to work in isolation. A point raised during the workshop was that the EC may have a role to play in ensuring that information on all alternative/Three Rs projects funded by individual DGs, is co-ordinated and available in one place; it is currently difficult to trace these Three Rs projects by other means. However, to ensure the dissemination of information on alternative methods, the Commission has already made a first step with the development of the comprehensive ECVAM database (DB-ALM), which offers public access to information on various aspects on alternatives to animal procedures $(2,3)$.

The EPAA workshop identified a gap. This was the absence of a process and/or organisation/institution supporting the post-validation and implementation of new methods, that would help ensure the rapid and widespread uptake of new alternative methods. The case studies presented at the workshop have clearly illustrated that what has been achieved so far was mainly due to the resource and perseverance of personal champions. This, however, is not a very efficient way forward for the future. The case studies also illustrated that long-term investment in the Three Rs is crucial to the support of Three $\mathrm{Rs}_{\mathrm{s}}$ methods - it was evident that, in all three case studies, the timescale from basic scientific concept to validated method was about 15 years. This not only comprises investment in research, but also in dissemination activities at various stages from research and development, to validation, regulatory acceptance, and implementation (see Figure 1).

Since dissemination plays an important role during the post-validation/implementation phase and for regulatory acceptance, the EPAA will seek synergy between the dissemination and validation and acceptance work strands, and will ensure that regulatory authorities are consulted.

\section{Acknowledgements}

The co-chairs of EPAA WG3 (Sally Robinson \& Jens Linge) would like to thank all of the participants for their contributions to the successful workshop. In addition, we would like to thank the following members of EPAA WG3, who helped with the organisation of the workshop, but were unable to attend: Janet Kelly (Covance Laboratories, Yorkshire, UK), Colin Dunn (Pfizer, Kent, UK) and Jan-Dirk SeilerHausmann (Henkel, Düsseldorf, Germany). The workshop was financed by the EPAA.

\section{Figure 1: Dissemination Circle for Three Rs methods}

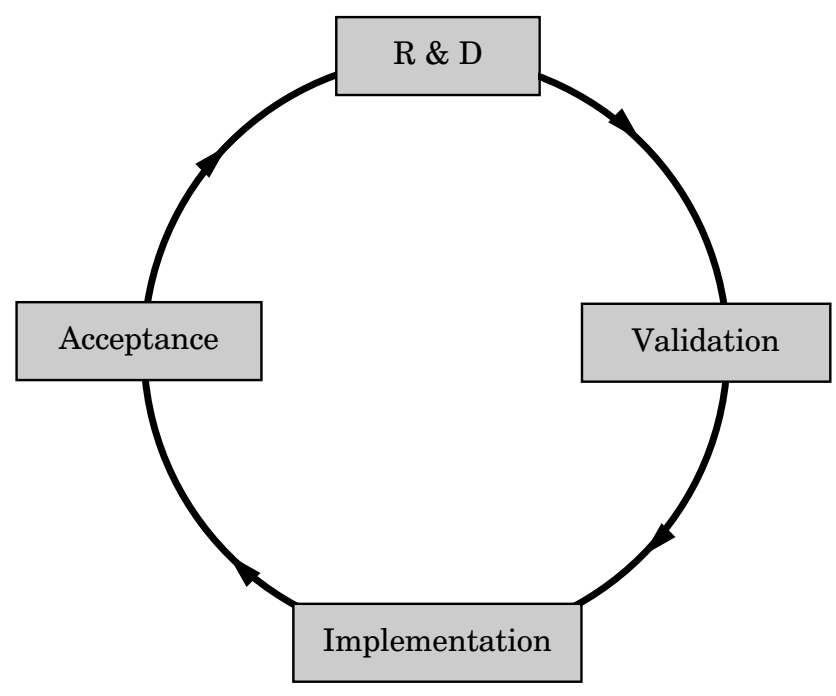

Dissemination activities should take place at various stages, in order to speed up the process. The collection of data involving the use of an already-accepted method might lead to further research and development, hence closing the circle. 


\section{References}

1. EPAA website: http://ec.europa.eu/enterprise/epaa/ (Accessed 01.04.08).

2. Russell, W.M.S. \& Burch, R.L. (1959). The Principles of Humane Experimental Technique, 238pp. London, UK: Methuen.

3. Anon. (1991). Establishment of a European Centre for the Validation of Alternative Methods (CEVMA). Communication from the Commission to the Council and the European Parliament, 29 October 1991. SEC(91)1794 final, 6pp. Brussels, Belgium: European Commission.

4. DB-ALM website: http://ecvam-dbalm.jrc.ec.europa. eu (Accessed 01.04.08).

5. OECD (2002). OECD Guideline for the Testing of Chemicals. No. 429. Skin Sensitisation: Local Lymph Node Assay, 7pp. Paris, France: Organisation for Economic Cooperation and Development. Available at: http://lysander. sourceoecd.org/vl $=7698543 / \mathrm{cl}=30 /$ $\mathrm{nw}=1 / \mathrm{rpsv} / \mathrm{cw} / \mathrm{vhosts} /$ oecdjournals/1607310x/v1n4/ contp1-1.htm (Accessed 13.08.08).

6. OECD (1992). OECD Guideline for the Testing of Chemicals. No. 406. Skin Sensitisation, 9pp. Paris, France: Organisation for Economic Cooperation and Development. Available at: http://lysander. sourceoecd. org $/ \mathrm{vl}=7698543 / \mathrm{cl}=30 / \mathrm{nw}=1 / \mathrm{rpsv} / \mathrm{cw} / \mathrm{vhosts} /$ oecdjournals/1607310x/v1n4/contp1-1.htm (Accessed 13.08.08).

7. Levin, J. \& Bang, F.B. (1964).The role of endotoxin in the extracellular coagulation of limulus blood. Bulletin of the Johns Hopkins Hospital 115, 265-274.

8. Anon. (1987). Guideline on Validation of the Limulus Amebocyte Lysate Test as an End-product Endotoxin Test for Human and Animal Parenteral Drugs, Biological Products and Medical Devices, 49pp. Rockville, MD, USA: Center for Drug Evaluation and Research, Food and Drug Administration. Available at: www.fda.gov/CbER/gdlns/lal.pdf (Accessed 14.08.08).
9. European Commission (2000). EU Directive 2000/33/ $E U$ for the 21st Amendment of Annex V of the EU Directive 86/906/EEC for classification and labelling of hazardous chemicals: Test Guideline B-41 "Phototoxicity - In vitro 3T3 NRU phototoxicity test". Official Journal of the European Communities L136, 98-107.

10. Spielmann, H., Balls, M., Dupuis, J., Pape, W.J.W., Pechovitch, G., de Silva, O., Holzhütter, H.G., Clothier, R., Desolle, P., Gerberick, F., Liebsch, M., Lovell, W.W., Maurer, T., Pfannenbecker, U., Potthast, J.M., Csato, M., Sladowski, D., Steiling, W. \& Brantom, P. (1998). The international EU/COLIPA in vitro phototoxicity validation study: results of Phase II (blind trial). Part 1: The 3T3 NRU phototoxicity test. Toxicology in Vitro 12, 305-327.

11. Spielmann, H., Balls, M., Dupuis, J., Pape, W.J.W., de Silva, O., Holzhütter, H.G., Gerberick, F., Liebsch, M., Lovell, W.W. \& Pfannenbecker, U. (1998). A study on UV filter chemicals from Annex VII of European Union Directive 76/768/EEC, in the in vitro 3T3 NRU phototoxicity test. ATLA 26, 679-708.

12. OECD (2004). OECD Guideline for the Testing of Chemicals. No. 432. In Vitro 3T3 NRU Phototoxicity Test, 15pp. Paris, France: Organisation for Economic Cooperation and Development. Available at: http://lysander. sourceoecd.org/vl $=7698543 / \mathrm{cl}=30 /$ nw $=1 / \mathrm{rpsv} / \mathrm{cw} /$ vhosts/oecdjournals/1607310x/v1n4/ contp1-1.htm (Accessed 13.08.08).

13. Robinson, S., Delongeas, J.L., Donald, E., Dreher, D., Festag, M., Kervyn, S., Lampo, A., Nahas, K., Nogues, V., Ockert, D., Quinn, K., Old, S., Pickersgill, N., Somers, K., Stark, C., Stei, P., Waterson, L. \& Chapman, K. (2008). A European pharmaceutical company initiative challenging the regulatory requirement for acute toxicity studies in pharmaceutical drug development. Regulatory Toxicology \& Pharmacology 50, 345-352. 